\title{
Circles, Spacers and Sutellites on the Riviera
}

from a Correspondent

TAKE about fifty scientists, isolate them on a small island an hour's journey from the mainland, reserve six hours a day for refreshment and it seems you have the prescription for a productive scientific meeting. This certainly was the case for the meeting held from May 5-9 at Port-Cros, supported by the Centre National de la Recherché Scientifique, and organized by Drs G. Bernardi, Paoletti and P. Slonimski.

The sessions on nuclear DNA were much the most controversial and the dominating issue was concerned with Callan's master-slave hypothesis. Dr R. J. Britten (Carnegie Institute) had set the scene by describing his well known observations on reassociation rates which show that a large fraction of the eukaryotic genome consists of repetitive sequences. But, as also emphasized by many other speakers, the properties of the reassociated duplexes indicate that the sequences are related but are not identical. This is directly contrary to the view of $\mathrm{Dr}$ C. A. Thomas (Harvard Medical School) who with brilliant advocacy sought to convince his audience that the formation of circles after the singlestranded ends of eukaryotic DNA have been exposed by exonuclease digestion provides evidence which favours the idea that much of DNA comprises exact multiple copies of genes. There are many technical questions involved, but it is probably fair to say that for one reason or another most of the participants remained unconvinced at the end of the meeting that exact copies had been demonstrated by the circle experiments.

A related issue was concerned with the interpretations of the kinetics of reassociation; this stemmed from the demonstration last year by $\operatorname{Dr}$ E. M. Southern (University of Edinburgh) that one satellite could best be described as a repeated hexamer into which a high proportion of mutations has been introduced. This description is very different from that predicted from the reassociation kinetics. Evidence which may resolve this discrepancy was presented by Dr B. J. McCarthy (University of Washington) who, in addition to discussing the separation of functional from non-functional chromatin, also described an inverse correlation between reassociation rates and the degree of mismatching of heteroduplex DNA. Southern argued on the basis of related experiments that a strong implication of the effect of mismatching would be that fractions which give ill-matched duplexes, such as the repetitive fraction from calf, could conceal a structural regularity based on short satellite-like sequences.

Two other less controversial theories of results were also discussed; both Drs D. D. Brown (Carnegie Institute of Washington) and M. Birnstiel (University of Edinburgh), in describing their results on gene organization, emphasized the curious properties of spacer DNA; in particular, it is now well established that although cistrons coding for ribosomal RNA are strongly conserved in evolution, spacer DNA is not. Indeed, Brown has shown that spacer sequences are apparently identical to each other in Xenopus laevis, but are considerably different from all those in $X$. mulleri. Drs F. Ritossa (Naples) and J. G. Gall (Yale University) considered the problem of differential amplification in Drosophila; it seems that although the proportion of ribosomal genes varies in the different bobbed mutants, the deficiency can be made good by subsequent gene amplification. Ribosomal genes and satellite DNA also seem to be amplified to different extents during the production of the giant chromosome.

In the mitochondrial field, the main interest centres round the production of petites by ethidium bromide, and in particular the variability in DNA sequence that can be found in different petite clones. Drs Slonimski and $\mathbf{H}$. Fukuhara (Gif-sur-Yvette) showed by hybridization experiments that some of the petite genes are amplified while others are lost, although the clearest evidence on amplification came from the tRNA cistrons studied by $\operatorname{Dr} \mathbf{M}$. Rabinowitz (Chicago). It seems from this work and that of Dr H. R. Mahler (Indiana University) that ethidium bromide can induce considerable sequence alteration and loss of mitochondrial DNA. But once established, the altered DNA is relatively stable in different clones although, as Slonimski emphasized, no petite strains can survive the rigours of a wild-type existence. This contrasts with the spacer and satellite DNAs in the nucleus and the situation in the normal mitochondria studied by Dr I. B. David (Baltimore), who has shown that in the two Xenopus species, the ribosomal cistrons in the mitochondria are conserved, but not the remainder which he thinks may have a spacer function.

Dr P. Borst (Amsterdam) and, particularly, Dr J. Vinograd (California Institute of Technology) were concerned with visualizing the normal processes of mitochondrial DNA replication. Vinograd has found that a small single-stranded piece of DNA is synthesized in a kind of blister in the mitochondrial supercoil, but it seems that replication cannot proceed further until a nick releases the strain induced by the partial unwinding.

Finally, Dr Francis Crick (MRC, Cambridge) accomplished the near miracle of presiding over a final discussion which genuinely stimulated the participants to reconsider and probe the earlier contributions. On the topic of mitochondria, this included a discussion of how a study of these organelles could contribute to molecular biology. It was felt that both the reasons why some functions were obligatorily specified by the mitochondrial genes and the aberrant DNA synthesis in petites could have general significance. On the topic of nuclear DNA, there was the opportunity of hearing Dr H. G. Callan (University of St Andrews) briefly review the cytogenetic evidence for his master-slave hypothesis, including the requirernent for sequence rectification, which probably takes place at every mitosis, because mutations occur as frequently in cultured cells as in whole organisms.

Thomas's circles, Southern's satellite sequences and Brown's spacers were all further probed and their interrelations discussed. This led Professor P. M. B. Walker (University of Edinburgh) to propose that preconceived notions should be discarded and it should be considered whether the genome could be composed of only a small fraction, perhaps less than 10 per cent, of genes coding for proteins, of which few could be exact copies; the remainder might have unknown or ill understood functions such as those of spacer and satellite DNA, or might be sequences evolved from them. This idea was supported by McCarthy who has found that nuclear RNA is much more divergent in related species than the polysomal message, but others perhaps found difficulty in accepting so large a fraction for which it is still impossible to demonstrate a proper function. In conclusion, Dr Crick said that he felt studies on the remaining genome were at a stage comparable to the period when ribosomal RNA was thought to carry the genetic message and all kinds of controversial new ideas were being canvassed. Clearly, the participants at least had every reason to thank the organizers, especially Dr Georgio Bernardi, for providing such a stimulating programme and environment for scientific discussion. 